\title{
Selective Detection of Backscattered Electrons in the Compound Lens Equipped UHR SEM
}

\author{
Petr Wandrol ${ }^{1}$, Radovan Vašina ${ }^{1}$, Adrian Sandu ${ }^{2}$, Ernst Jan Vesseur ${ }^{2}$ and Lubomír Tůma ${ }^{1}$. \\ 1. FEI Company, Brno, Czech Republic \\ 2. FEI Company, Eindhoven, the Netherlands
}

Detection of signal electrons belongs amongst the key parameters of the Scanning Electron Microscope (SEM). The traditional approach is the ETD detector for secondary electrons (SE) and a below-the-lens detector for backscattered electrons (BSE). State-of-the-art SEMs can be equipped with up to three inlens detectors capable of collecting both the SE and BSE signal. Moreover, the possibility to sort electrons according to their energies and/or emission angles is becoming common. Such a selective detection is usually done by influencing the SE or BSE trajectories by electric or magnetic fields. This paper introduces compound-lens-controlled energy selective detection of BSE on a new FEI SEM.

The compound final lens combines the magnetic final lens inside the pole piece (ML1), the immersion magnetic lens (ML2) and the electrostatic lens (EL1) formed by the potential at the T1 detector (Fig. 1a). Main function of the compound final lens is to focus the primary electron beam to the sample. However, independent control of these three lenses enables us to use ML1 and EL1 to focus the primary beam and the ML2 to affect the trajectories of signal electrons. The ML2 behaves as a chromatic sensitive lens which focuses the high-loss BSE into the aperture of the annular T1 detector. The low-loss BSE are less sensitive to the ML2 magnetic field and reach the T1 detector (Fig. 1b). SE also pass through the aperture in the T1 and are collected further in the column by the T2 and T3 detectors.

The main goal of the energy selective BSE detection is the enhancement of the compositional contrast. The BSE signal typically contains both the high- and the low-loss fractions. The BSE coefficient $(\eta)$ is little dependent on the atomic number $(Z)$ for the high-loss BSE, while it is strongly dependent in the case of the low-loss BSE. Therefore, in order to enhance the compositional contrast, low-loss BSE need to be selectively detected. This is exactly the mechanism behind the contrast enhancement with the compound lens. Besides the BSE filtration, the magnetic field of the ML2 decreases the diameter of the primary beam and thus improves the resolution. This enables us to acquire compositional contrast images in high resolution, such as the Pd nano-particles on the $\mathrm{CeO}_{2}$ matrix in Fig. 2a [2]. Another important improvement is the collection efficiency of the T1 BSE detector. Thanks to its design and position, the T1 detector covers a large BSE emission angle and provides high signal to noise ratio even at low probe currents. Naturally, BSE filtering cuts off a significant part of the T1 signal. However, the T1 detector keeps providing low noise images at probe currents down to $25 \mathrm{pA}$ and less. The possibility to work at low probe currents together with the energy selective BSE detection enables charge-free imaging of non-conductive samples. The high-loss BSE carrying the charge information are filtered out so they don't contribute to the T1 detector image. Collection of low-loss BSE at probe current of 25pA produces charge free images of insulators, such as polymer covered by silver particles in Fig 2b [3]. An image acquired with the complete BSE energy spectrum is included for comparison (Fig 2c).

In conclusion, energy selective detection of backscattered electrons using a new compound electrostaticmagnetic immersion lens allows for enhanced resolution, enhanced compositional contrast and chargefree imaging of insulators, while maintaining high signal to noise at probe currents as low as $25 \mathrm{pA}$. 
References:

[1] $\mathrm{PdCeO} 2$ sample courtesy of Dr. Alessandro Lavacchi, CNR ICCOM

[2] Ag particles on polymer sample courtesy of the TE01020218 project team, Membrain

[3] The authors acknowledge funding from the Technology Agency of the Czech Republic, TE01020118
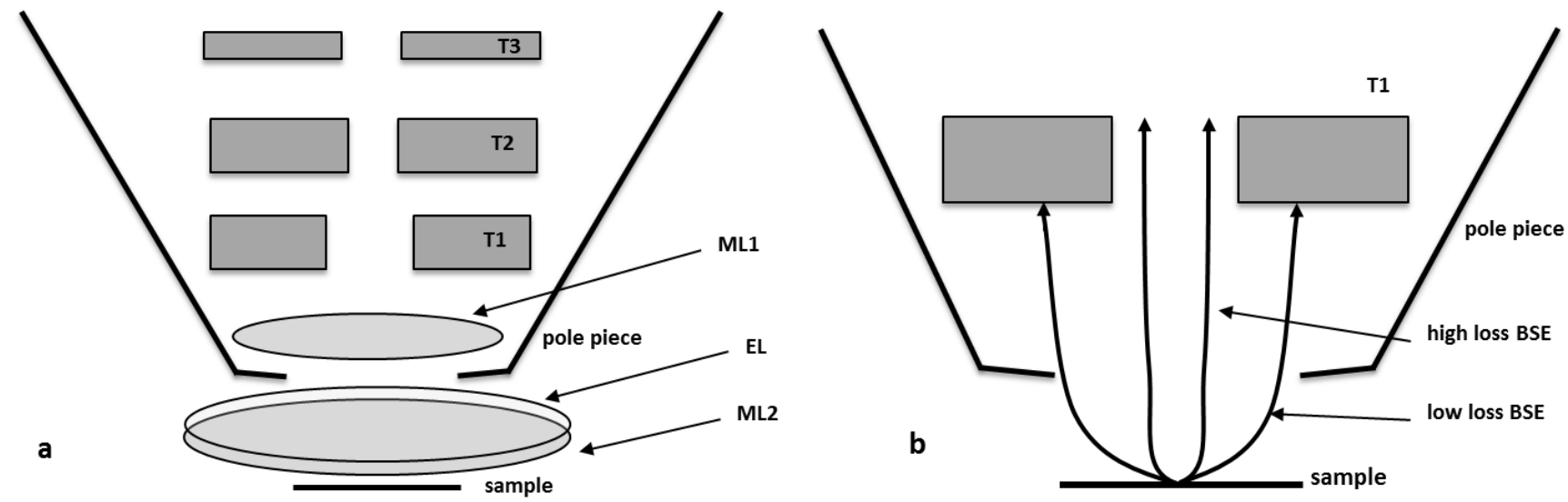

Figure 1. Scheme of the compound final lens (a) and trajectories of backscattered electrons influenced by the magnetic field of the ML2 (b).
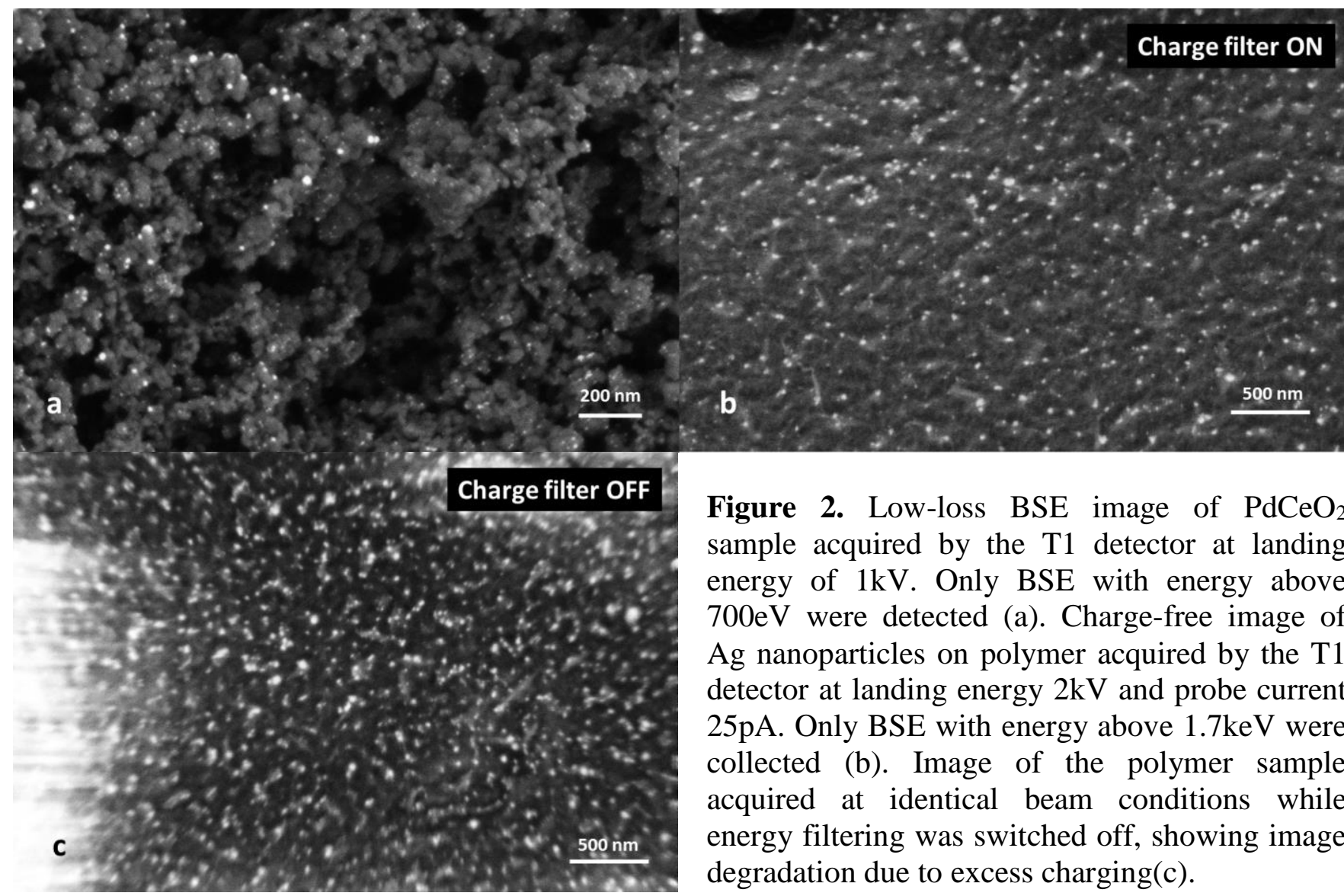

Figure 2. Low-loss $\mathrm{BSE}$ image of $\mathrm{PdCeO}_{2}$ sample acquired by the $\mathrm{T} 1$ detector at landing energy of $1 \mathrm{kV}$. Only BSE with energy above $700 \mathrm{eV}$ were detected (a). Charge-free image of $\mathrm{Ag}$ nanoparticles on polymer acquired by the $\mathrm{T} 1$ detector at landing energy $2 \mathrm{kV}$ and probe current $25 \mathrm{pA}$. Only BSE with energy above $1.7 \mathrm{keV}$ were collected (b). Image of the polymer sample acquired at identical beam conditions while energy filtering was switched off, showing image degradation due to excess charging(c). 